\title{
NCCN Conference Addressed Value-Based Healthcare Models, Payer Perspectives, New NCCN Guidelines, and Other Updates in Cancer Care
}

NCCN hosted the 23rd NCCN Annual Conference to address some of the biggest emerging issues in oncology currently, including receiving cancer care in value-based healthcare models, managed care, and CMS reimbursement. The NCCN conference also highlighted new treatment advances in immunotherapy, genetic testing, and the latest developments in breast cancer care. The conference took place March 22-24, at Rosen Shingle Creek in Orlando, Florida.

"The NCCN 23rd Annual Conference is designed to address important educational needs to help cancer care providers improve the quality, effectiveness, and efficiency of cancer care so that patients can live better lives," said Robert W. Carlson, MD, Chief Executive Officer, NCCN. "The NCCN Annual Conference focuses on the practical management of patients with cancer, provides updates of NCCN Guidelines, and follows new trends for treatment. With the increased pace of cancer research, large number of disease sites and biologically distinct subtypes, emergence of new diagnostic markers and multigene assays, and expansion of new treatment options, there is tremendous need for clinicians to maintain and continually add to their knowledge of scientific concepts and how to apply them in the everyday practice setting."

The keynote address featured Ron Kline, MD, Medical Officer, Patient Care Models Group; Center for Medicare and Medicaid Innovation (CMMI), Centers for Medicare \& Medicaid Services (CMS); and Lee N. Newcomer, MD, MHA, former Senior Vice President, Oncology and Genetics, UnitedHealthcare, offering payers' perspectives on how cancer care in America is evolving.

Following the keynote addresses was a roundtable on "Emerging Issues in Oncology A Multidisciplinary Discussion on Delivering and Receiving Cancer Care in Value-Based Healthcare Models." The roundtable was moderated by Clifford Goodman, PhD, Senior Vice President, The Lewin Group, and featured the following panelists:

- Travis H. Bray, PhD, Founder, Hereditary Colon Cancer Foundation

- Randy Burkholder, Vice President of Policy and Research, PhRMA

- Ron Kline, MD, Medical Officer, Patient Care Models Group, CMMI, CMS

- Daniel P. Mirda, MD, President, Association of Northern California Oncologists (ANCO)

- Michael N. Neuss, MD, Chief Medical Officer, Vanderbilt-Ingram Cancer Center

- Lee N. Newcomer, MD, MHA, former Senior Vice President, Oncology and Genetics, UnitedHealthcare

- Bhuvana Sagar, MD, National Medical Director, Cigna

Three new NCCN Clinical Practice Guidelines for Oncology (NCCN Guidelines) were presented during the conference, including:

- Management of Immunotherapy-Related Toxicities

- Cancer in People Living With HIV

- Uveal Melanoma

There were also presentations featuring updates on treatments for the following types of cancer:

- Acute Lymphoblastic Leukemia

- Acute Myeloid Leukemia

- Breast

- Chronic Myeloid Leukemia

- Colorectal

- Head and Neck

- Kidney 
- Lung

- Melanoma

- Multiple Myeloma

- Ovarian

- Prostate

- Urothelial Carcinoma

New developments in supportive care were also addressed in various sessions. The agenda included:

- Educational Presentations: More than 20 educational sessions provided an overview of the current state-of-the-art in oncology care across multiple cancer sites.

- General Poster Sessions: Approved research abstracts offered new data in the areas of clinical and preclinical oncology, epidemiology, genomics, clinical practice guidelines, quality improvement, outcomes, and information technology.

- Young Investigator Presentations: Young Investigators who received research grants from the NCCN Foundation's Young Investigator Awards program presented data about their research.

\section{For Nurses:}

The NCCN 2018 Oncology Nursing Program took place on Wednesday, March 21. It provided oncology nurses with comprehensive and clinically relevant information to optimize patient education and care focused around the following topics:

- Improving Quality of Care for Lesbian, Gay, Bisexual, and Transgender (LGBT) Patients

- Substance Use in Patients with Cancer; Optimizing Oral Therapy in Kidney Cancer

- Management of Cardiovascular Toxicities Associated with TKI Therapy

- Management of Hypersensitivity Reactions to Systemic Therapy

- Safe Handling of Hazardous Drugs

- Implementing a CAR T-Cell Therapy Program

\section{For Industry:}

The NCCN Academy for Excellence \& Leadership in Oncology offered pharmaceutical and biotechnology professionals a rare opportunity to view the oncology space from the provider and payer perspective. The program took place Wednesday, March 21, from 7:30 AM to 2:30 PM. Modules included:

- Discovery and Innovation: Redefining Cancer Care, Quality, and Value

- Meet the NCCN Guidelines Panel Experts

- Supporting the Patient Journey: Advances and Best Practices in Supportive Care, Survivorship and Patient Advocacy

To view the full conference agenda, visit NCCN.org/conference. Join the conversation online with the hashtag \#NCCNac18. 